\title{
A Structural Equation Model of Social Support, Stress and Depression in Pregnant Women During Epidemic Period
}

\author{
Yi Liang ${ }^{1}$, Shengfeng Dong ${ }^{2}$, Liling Zhu ${ }^{3}$, Danqing Zhao ${ }^{1}$, and Liming Shen ${ }^{4}$ \\ ${ }^{1}$ The Affiliated Hospital of Guizhou Medical University \\ ${ }^{2}$ Fifth Affiliated Hospital of Zunyi Medical University \\ ${ }^{3}$ Chinese University of Hong Kong RCT \\ ${ }^{4}$ Shenzhen University
}

December 1, 2020

\begin{abstract}
Objective: To investigate the connections among social support, stress, and depression. Design: Cross-sectional study. Setting: Guizhou Province in China. Population or Sample: An aggregate of 1,056 expectant ladies, had finished our questionnaire during pregnancy from March to April in 2020. Methods: The Edinburgh prenatal sadness scale, an independent pressure scale, and social support scale evaluated the downturn, stress, and social support of pregnant people during the pestilence. An auxiliary condition model was utilized to examine the immediate and aberrant connection between social support and prenatal misery. Main Outcome Measures: Incidence of depression. Results: During the pandemic time, $73.01 \%$ of pregnant ladies experienced prenatal misery. The model is suitable (chi-square $=11.96, \mathrm{CFI}=0.97, \mathrm{RMSEA}=0.07, \mathrm{RMR}=0.03$ ). The auxiliary condition model indicated that the immediate pathway of social support to depression was critical (normalized pathway coefficient $=-$ 0.34), and the aberrant pathway of stress to depression via social support was additionally huge (normalized pathway coefficient $=0.50)$. Stress partially intercedes the connection between social support and depression. Conclusion: Our discoveries posit that social support is related to an expanded danger of depression. Stress is decidedly corresponding to depression and assumes an interceding position between social support and stress. Thusly, directed mediation ought to be completed to lessen the depression of pregnant ladies and improve their psychological wellness status. Keywords: stress, depression, social support, structural equation model
\end{abstract}

A structural equation model of social support, stress and depression in pregnant women during epidemic period: a cross-sectional study

\section{Yi Liang ${ }^{1}$, Shengfeng Dong ${ }^{2}$, Liling Zhu ${ }^{3}$, Danqing Zhao ${ }^{*}$, Liming Shen ${ }^{*}$}

${ }^{1}$ Department of Clinical Nutrition, Affiliated Hospital of Guizhou Medical University, 28 Guiyi Street Guiyang 550004, P.R. China

${ }^{2}$ Department of Obstetrics and Gynecology, Affiliated Hospital of Zunyi Medical University, Zunyi 563099, P.R. China

3 The Jockey Club School of Public Health and Primary Care, Chinese University of HongKong, HongKong 999077, P.R. China

${ }^{4}$ Department of Obstetrics and Gynecology, Affiliated Hospital of Guizhou Medical University, Guiyang 550004, P.R. China

${ }^{5}$ College of Life Science and Oceanography, Brain Disease and Big Data Research Institute, Shenzhen University, Shenzhen 518060, P.R. China 


\section{* Correspondence:}

Liming Shen

E-mail: slm@szu.edu.cn

Danqing Zhao

E-mail: 1070627325@qq.com

Running head: social support, stresss and depression

ABSTRACT

Objective : To investigate the connections among social support, stress, and depression. Design: Crosssectional study.

Setting: Guizhou Province in China.

Population or Sample: An aggregate of 1,056 expectant ladies, had finished our questionnaire during pregnancy from March to April in 2020.

Methods: The Edinburgh prenatal sadness scale, an independent pressure scale, and social support scale evaluated the downturn, stress, and social support of pregnant people during the pestilence. An auxiliary condition model was utilized to examine the immediate and aberrant connection between social support and prenatal misery.

Main Outcome Measures: Incidence of depression.

Results : During the pandemic time, $73.01 \%$ of pregnant ladies experienced prenatal misery. The model is suitable (chi-square $=11.96, \mathrm{CFI}=0.97, \mathrm{RMSEA}=0.07, \mathrm{RMR}=0.03)$. The auxiliary condition model indicated that the immediate pathway of social support to depression was critical (normalized pathway coefficient $=-0.34$ ), and the aberrant pathway of stress to depression via social support was additionally huge (normalized pathway coefficient $=0.50$ ). Stress partially intercedes the connection between social support and depression. Conclusion : Our discoveries posit that social support is related to an expanded danger of depression. Stress is decidedly corresponding to depression and assumes an interceding position between social support and stress. Thusly, directed mediation ought to be completed to lessen the depression of pregnant ladies and improve their psychological wellness status.

Keywords: stress, depression, social support, structural equation model

\section{INTRODUCTION}

Coronavirus disease 2019 (COVID-19), birthed by the 2019 unusual Covid, is an exceptionally infectious illness. It has now become an overall pandemic ${ }^{1}$. WHO has proclaimed the current pandemic a worldwide general wellbeing crisis. Internationally, starting on 26 October 2020, there have been 42,966,344 affirmed instances of COVID-19, including 1,152,604 demises, conveyed to WHO. Because of the incredible endeavors of the World Health Organization and worldwide scourge anticipation, the infection has been successfully controlled in certain nations, yet the global circumstance is yet not confident. Simultaneously, numerous other wellbeing challenges remain, including the mental pressure, mental weight, social support, and several difficulties caused the epidemic ${ }^{2,3}$

Depression is a typical difficulty of pregnancy, and its indications range from mellow despondency to extreme depression, which is regularly joined by dread, nervousness, a sleeping disorder, and impassion or antagonism towards the spouse and additionally newborn ${ }^{4}$. For moms, depression during pregnancy is not just connected with postnatal complications ${ }^{5-7}$; it is additionally identified with a higher danger of gestational diabetes mellitus (GDM), hypertension, and preeclampsia ${ }^{8-11}$. The transient impacts of a mother's downturn on 
the infant are more serious dangers of untimely birth, low birth weight, and development impediment. Enthusiastic and social issues are a portion of the drawn-out negative results for offspring of discouraged mothers ${ }^{12}$, similar to cognitive development impairment ${ }^{13,}{ }^{14}$, attention deficit disorder ${ }^{15},{ }^{16}$. Based on the huge impacts of prenatal depression on the medium and long term wellbeing of both mother and child, it is critical to distinguish adjustable danger elements that add to prenatal depression circumvention in the Chinese populace.

Exploration reveals that the pestilence circumstance of COVID-19 can influence the emotional well-being of the populace. $58 \%$ of the overall populace feels that COVID-19 has a moderate and serious mental effect (16.5\%, moderate and extreme discouragement; $28.8 \%$, moderate and extreme tension; $8.1 \%$, moderate and extreme pressure level $)^{17}$. This extra mental weight may irritate the enduring of pregnant ladies, particularly the individuals who need emotionally supportive networks, because powerful social emotionally supportive networks may ease these issues ${ }^{18}$.

Investigations have demonstrated that prenatal depression in expectant women is firmly identified with pressure and social support ${ }^{19-21}$, however, a couple of investigations focus on the mediating impact of pressure between social support and sorrow. Simultaneously, concerning the worldwide pandemic, the mental state of expectant ladies has changed definitely. There is no investigation on the connection between prenatal sorrow, stress, and social support with regards to the pandemic. In this examination, we utilized a survey star to gather the gauge information of expectant ladies during the pandemic time frame to investigate the connection between ladies' prenatal gloom, stress, and social support, to give a more focus on mental mediation and an arrangement of social support for pregnant ladies later on.

\section{MATERIAL AND METHODS}

\section{Study sample}

\section{Study site}

The subjects were expectant ladies enrolled in Guizhou Province from March to April 2020. Utilizing a cross-sectional examination by stratified sampling strategy, Guizhou Province was partitioned into 7 strata as indicated by Guiyang, Qiannan Prefecture, Anshun, Zunyi, Bijie, Tongren, and Liupanshui. Two public medical clinics were randomly chosen inside each metropolitan zone, while 2-3 public medical clinics were arbitrarily chosen inside each countryside zone.

\section{Participants and sampling}

Incorporation models for the examination were expectant ladies with clinical cards, prenatal registration arrangements, and who ready to partake in the investigation. The expectant ladies who declined to finish the surveys because of mental, actual ailment, or different reasons (for example neglecting to have sufficient opportunity) were expunged.

An aggregate of 1,056 expectant ladies, having set up a clinical card for a prenatal registration during pregnancy from March to April in 2020 in Guizhou Province, China, was selected for the examination.

\section{Questionnaire}

The educated assessors and nearby network medical attendants requested each qualified pregnant lady to fill in a self-regulated survey to gather segment attributes, pregnancy data (last menstrualcycle, pregnancy recurrence, the record of antagonistic pregnancy, pregnancy difficulties, etc.), and information disposition/conduct poll for COVID-19. A few members, likewise, finished the depression/perceived pressure surveys during the pandemic of COVID-19, which were set up on an online stage using www.wjx.cn in mainland China, and members were approached to finish polls through the Internet. The ethics council of the Affiliated Hospital of Guizhou Medical University endorsed this examination. All the strategies utilized in this examination were led as per applicable rules and guidelines.

\section{Instruments}




\section{Edinburgh Prenatal Depression Scale (EPDS)}

The Edinburgh Prenatal Depression Scale was utilized to assess depression during pregnancy. The device has been broadly utilized in 35 nations and has been approved in China. There are ten items in the Edinburgh Prenatal Depression Scale. The score scope of each piece is 0-3, yet various scores of individual items convey various implications. The all out score range is 0-30. EPDS is a self-rating scale, which takes around 5 minutes to finish. The basic estimation of the Chinese form of Edinburgh Prenatal Depression Scale utilized in this investigation is 9.5, that is, the point at which the EPDS score comes to above 9.5, it shows depression during pregnancy.

\section{Perceived Stress Scale (PSS)}

PSS was utilized to become more acquainted with the member's emotions and contemplations inside the one-month timeframe. The scale incorporates 14 items, each of which was evaluated by varying frequencies, specifically: never, periodically, at times, frequently, and consistently. Scores shift as per different frequencies. The absolute scores of all things were used to survey stress level.

\section{Multidimensional Scale of Perceived Social Support (MSPSS)}

MSPSS was designed to evaluate the level of emotional observed social support from family, companions, and other significant connections. The scale, with an aggregate of 12 things, includes three sub-scales, to be specific family support, companion backing, and fundamental relationship support. Various degrees of understanding and difference were set in the scale, to be totally disagree, strongly disagree, partially disagree, uncertain, partially agree, strongly agree, and totally agree, every one of which was allotted to various scores.

\section{Data analysis}

Concluded information was gathered and transferred from the online stage referenced previously. With data imported and checked for culmination, SAS9.3 was used for datacalculation, and Amos 22.0 was utilized to construct the organizational condition model. Descriptive statistics were accounted for on socio-segment qualities, social support, depression, and feeling of anxiety. Mean \pm standard deviation (SD) was utilized to portray regularlyappropriated constant factors, median (interquartile range, IQR) for skewed-circulated consistent factors, and frequencies (percentage) for sectionalized factors. In this investigation, social support, stress, and depression scores were unceasing factors, that did not adjust to an ordinary circulation, hence, Spearman correlation examination was utilized to test the connection.

This examination constructed a structural equation model of social support, stress, and depression and tried the intervening part of pressure between social support and depression. Confirmatory Factor Analysis (CFA) assessed the decency of fit. Through a satisfactory measurement model,a Structural Equation Model (SEM) was utilized to assess the theoretical connections among social support, stress, and depression. In the bivariate examination, the sociodemographic attributes fundamentally connected with depression were controlled as covariates in SEM. Since social support, stress, and depression do not comply with typical appropriation, we utilized the powerful most extreme probability assessment (MLR) to gauge the boundaries of the basic condition model. In the event that $\mathrm{P}<0.05$ was gotten by a respective test, the boundaries were viewed as factually huge. Chi-square/DF, Comparative Fit File (CFI), Root Mean Square Error of Approximation (RMSEA), and Standard Root Mean Square Residual (SRMR) were utilized to assess the fitting between the estimation model and the auxiliary model. On the off chance that the proportion of chi-square/DF $<$ 3.0, CFI [?] 0.95, RMSEA [?] 0.06, SRMR $<0.08$, the model fitting is regarded as acceptable.

\section{RESULTS}

\section{General Characteristics}

Maternal qualities are revealed in Table 1. As per the Edinburgh Postpartum Depression Scale, 771 expectant ladies were determined to have depression. The predominance of prenatal depression was $73.01 \%$. The age of the respondents was 16-50 years of age, however, most were somewhere in the range of 16 and 40 years of age. $58.81 \%$ of expectant ladies lived in urban communities during the pestilence time frame. $62.32 \%$ of expectant 
ladies had advanced education levels (college or above), $50.57 \%$ were primiparas, $54.55 \%$ had family pay over 5000 yuan, and the expectant ladies in ahead of schedule, center, and late pregnancy represented $24.43 \%$, $45.27 \%$, and $30.30 \%$, separately. The frequency of pregnancy inconveniences was $19.89 \%$.

Contrasted and the undepressed set, the dejected gathering had a higher extent of more aged women, advanced education levels, higher family pay, and were bound to live in the field during the pandemic time frame.

\section{Correlations among depression, social support, and stress}

Table 2 demonstrates the relationship between depression, social support, and stress. Relationship examination indicated that social support was adversely associated with depression, while stress decidedly corresponded with depression. The Spearman connection coefficients were - $0.40(\mathrm{P}<0.01)$ and $0.27(\mathrm{P}<$ 0.01 ), respectively.

The immediate route from social support to depression was substantial (normalized pathway coefficient $=$ - 0.34, $\mathrm{P}<0.01$ ), and the ancillary pathway from stress to depression was additionally huge (normalized pathway coefficient $=0.50, \mathrm{P}<0.01)$.

\section{Measurement model}

The CFA demonstrated that the calculation archetypical produced a suitable model (Chi-square value $=11.96$, Degrees of Freedom $(\mathrm{DF})=9, P<0.01, \mathrm{CFI}=0.97, \mathrm{RMSEA}=0.07, \mathrm{SRMR}=0.03)$. Every element loadings stood at $P<0.01$ level. Outcome recalibrated element loads are displayed in Fig. 1.

\section{Direct and indirect effects of structural models}

The straight route from social support to depression was substantial (recalibrated route coefficient $=-0.34$, $P<0.01$ ), and the ancillary route from social support to depression also displayed a noteworthy figure (recalibrated route coefficient $=-0.09 \times 0.50=-0.45, P<0.01$ ). The outcome reveals that the recalibration impact of the ancillary route resembles the straight pathway (- $0.34 \mathrm{vs.}-0.45)$ (table 3 and table 4$)$. The SEM prototypical is presented in Fig. 1.

\section{DISCUSSION}

From this investigation, Structural Equation Model (SEM) was utilized to examine the correlation among social support, stress, and prepartum depression. The outcome revealed that a positive link exists between stress and prepartum depression, while there is an adverse relationship between social support and prepartum depression.

Our investigation reveals that the occurrence of prenatal depression is as high as $73.01 \%$ during the pandemic timeframe, which is a lot higher than that during the non-plague state ${ }^{22,23}$. This might be identified with the eruption of the pandemic and the exceptional time of pregnancy ${ }^{24}$. Moreover, we establish that the high-hazard elements of prenatal depression, for example, older age, might be identified with the higher physical and mental weight of more seasoned pregnant ladies than youthful pregnant ladies, which is reliable with Enayati's study ${ }^{25}$. Furthermore, expectant ladies in the city where the scourge happened are bound to have prenatal depression contrasted with those in country regions with less admittance to data about the pandemic. This might be connected in light of the fact that those in metropolitan zones are outfitted with refreshed data about the pandemic and would in general accept that the danger of getting tainted is higher in metropolitan regions than those in-country ones with less populace development. Likewise, similar to Yeon's investigation, this examination proposes that the higher the instructive level and the higher the family pay one has, the higher the danger is for prenatalprenatal depression, which might be identified with the more noteworthy tension on mental workers ${ }^{26}$.

There exist several affecting elements on prenatal depression in expectant ladies, among which social support and weight assume significant parts. The examination reveals the members would in general apply a lot of mental weight and philosophical weight on themselves, which place themselves into predicaments ${ }^{27}$. 
Expectant women have fewer occasions to go out during the pandemic timeframe, and going out will expand the danger of presentation to COVID-19. There is less up close and personal correspondence with loved ones, causing a tough and uneasy communication of their feelings. Comparable circumstances frequently lead to inordinate mental and philosophical tension on expectant ladies, which will negatively affect the two people and society in the long haul. Steady with the finish of this investigation, Zou's examination shows that great social support can improve some burdensome side effects, for example, the absence of inspiration and misery of life ${ }^{28}$. Accordingly, it is important to fortify family upholds, companion backing, and network upholds for expectant ladies during the plague time frame. Being instructed in emotional well-being and effectively taking an interest in mental guiding for pregnant ladies during the plague time frame will lessen their nervousness and improve their depression indications.

Simultaneously, this investigation likewise established that pressure has a fractional interceding impact between social support and depression. Expectantmothers during the pandemic time frame, from one viewpoint, bear the weight of pregnancy, then again, they are upset by the scourge, subsequently, they are powerless against emerging ongoing pressure ${ }^{29}, 30$. Exploration indicates that pressure builds the danger of depression, which thusly prompts different mental issues. Numerous investigations have focused on the impact of weight on prenatal depression manifestations, recommending that focused intercession ought to be completed to ease pregnant ladies stress ${ }^{31}, 32$. Studies have affirmed that prenatal pressure the board courses can successfully lessen expectant mothers' pressure, subsequently diminishing expectant mothers' depression, however, there are a couple of related examinations in Chinaa ${ }^{31,32}$. Later on, these researchers may deliberate adding the substance of stress alleviation into the day-by-day management of expectant mothers and consistently complete pressure the board courses to assist pregnant ladies with prenatal depression manifestations to improve their psychological wellness.

This examination is the initial attempt to develop a structural condition model of social support, stress, and depression in expectant mothers during the pandemic timeframe. This examination established that the expansion in social support in this populace would decrease the frequency of depression. Simultaneously, the expansion of social support will likewise lessen the mental weight of expectant mothers, which will in anancillarymanner lead to a decrease of prenatal depression. The intervening function of pressure between social support and depression merits consideration. Through exposure, schooling, and social support, diminishing tension on expectant mothershelps decrease the depression of expectant mothers during the scourge time frame.

\section{Conclusion}

The psychological well-being of expectant mothers in the pandemic era in the Guizhou area is critical. Concerning patients with prepartum depression indications, lessening the pressure, and upscaling the social support will aid in alleviating their depression.

\section{Disclosure of interests}

None of the authors have any personal or financial conflicts of interest.

\section{Contribution to authorship}

Y.L. performed the analyses and wrote the manuscript. S.D., L.Z., coordinated the study centers, L.S. and D.Z. supervised the analyses, and D.Z. conceived the project. All authors critically reviewed the manuscript for important intellectual content.

\section{Details of ethics approval}

This article does not deal with ethical issues.

\section{Funding}

This study was financially supported by National Natural Science Foundation of China (Grant Nos. 81960284, 31870825), and Shenzhen-Hong Kong Institute of Brain Science-Shenzhen Fundamental Research 
Institutions, 2019SHIBS0003.

\section{Acknowledgements}

All participates and their families in the study are gratefully acknowledged. We also thank all colleagues working in the study for their continuing valuable help.

\section{REFERENCES}

1. Swerdlow DL, Finelli L, Lipsitch M. Epidemiology of Covid-19. Reply. N Engl J Med. 2020; 382:1869-70.

2. Pfefferbaum B, North CS. Mental Health and the Covid-19 Pandemic. N Engl J Med. 2020.

3. Liu Z, Wu J, Shi X, Ma Y, Ma X, Teng Z, et al. Mental Health Status of Healthcare Workers in China for COVID-19 Epidemic. Ann Glob Health. 2020; 86:128.

4. Committee on Obstetric P. The American College of Obstetricians and Gynecologists Committee Opinion no. 630. Screening for perinatal depression. Obstet Gynecol. 2015; 125:1268-71.

5. Munoz RF. Prevent depression in pregnancy to boost all mental health. Nature. 2019; 574:631-3.

6. Strohmaier S, Devore EE, Vetter C, Eliassen AH, Rosner B, Okereke OI, et al. Night shift work before and during pregnancy in relation to depression and anxiety in adolescent and young adult offspring. Eur J Epidemiol. 2019; 34:625-35.

7. Rejno G, Lundholm C, Oberg S, Lichtenstein P, Larsson H, D'Onofrio B, et al. Maternal anxiety, depression and asthma and adverse pregnancy outcomes - a population based study. Sci Rep. 2019; 9:13101.

8. Bandoli G, Chambers CD. Autoimmune conditions and comorbid depression in pregnancy: examining the risk of preterm birth and preeclampsia. J Perinatol. 2017; 37:1082-7.

9. Abedian Z, Soltani N, Mokhber N, Esmaily H. Depression and anxiety in pregnancy and postpartum in women with mild and severe preeclampsia. Iran J Nurs Midwifery Res. 2015; 20:454-9.

10. Arafa A, Dong JY. Depression and risk of gestational diabetes: A meta-analysis of cohort studies. Diabetes Res Clin Pract. 2019; 156:107826.

11. Hinkle SN, Buck Louis GM, Rawal S, Zhu Y, Albert PS, Zhang C. A longitudinal study of depression and gestational diabetes in pregnancy and the postpartum period. Diabetologia. 2016; 59:2594-602.

12. Leis JA, Heron J, Stuart EA, Mendelson T. Associations between maternal mental health and child emotional and behavioral problems: does prenatal mental health matter? J Abnorm Child Psychol. 2014; 42:161-71.

13. Bleker LS, Milgrom J, Gemmill AW, Roseboom TJ, de Rooij SR. A 7-year follow-up of antenatal depression treatment with cognitive behavioral therapy: A case report of maternal and child outcomes. SAGE Open Med Case Rep. 2019; 7:2050313X19841463.

14. Jensen SK, Dumontheil I, Barker ED. Developmental inter-relations between early maternal depression, contextual risks, and interpersonal stress, and their effect on later child cognitive functioning. Depress Anxiety. 2014; 31:599-607.

15. Sfelinioti S, Livaditis M. Association of maternal depression with children's attention deficit hyperactivity disorder. Psychiatriki. 2017; 28:251-8.

16. Leijten P, Scott S, Landau S, Harris V, Mann J, Hutchings J, et al. Individual Participant Data MetaAnalysis: Impact of Conduct Problem Severity, Comorbid Attention-Deficit/Hyperactivity Disorder and Emotional Problems, and Maternal Depression on Parenting Program Effects. J Am Acad Child Adolesc Psychiatry. 2020. 
17. Smith K, Ostinelli E, Cipriani A. Covid-19 and mental health: a transformational opportunity to apply an evidence-based approach to clinical practice and research. Evid Based Ment Health. 2020; 23:45-6.

18. Salehi L, Rahimzadeh M, Molaei E, Zaheri H, Esmaelzadeh-Saeieh S. The relationship among fear and anxiety of COVID-19, pregnancy experience, and mental health disorder in pregnant women: A structural equation model. Brain Behav. 2020:e01835.

19. Xie RH, He G, Koszycki D, Walker M, Wen SW. Prenatal social support, postnatal social support, and postpartum depression. Ann Epidemiol. 2009; 19:637-43.

20. Nylen KJ, O'Hara MW, Engeldinger J. Perceived social support interacts with prenatal depression to predict birth outcomes. J Behav Med. 2013; 36:427-40.

21. Goldstein JM, Hale T, Foster SL, Tobet SA, Handa RJ. Sex differences in major depression and comorbidity of cardiometabolic disorders: impact of prenatal stress and immune exposures. Neuropsychopharmacology. 2019; 44:59-70.

22. Yu Y, Zhu X, Xu H, Hu Z, Zhou W, Zheng B, et al. Prevalence of depression symptoms and its influencing factors among pregnant women in late pregnancy in urban areas of Hengyang City, Hunan Province, China: a cross-sectional study. BMJ Open. 2020; 10:e038511.

23. Yu M, Gong W, Taylor B, Cai Y, Xu DR. Coping Styles in Pregnancy, Their Demographic and Psychological Influences, and Their Association with Postpartum Depression: A Longitudinal Study of Women in China. Int J Environ Res Public Health. 2020; 17.

24. Samrah SM, Al-Mistarehi AH, Aleshawi AJ, Khasawneh AG, Momany SM, Momany BS, et al. Depression and Coping Among COVID-19-Infected Individuals After 10 Days of Mandatory in-Hospital Quarantine, Irbid, Jordan. Psychol Res Behav Manag. 2020; 13:823-30.

25. Enayati M, Solati J, Hosseini MH, Shahi HR, Saki G, Salari AA. Maternal infection during late pregnancy increases anxiety- and depression-like behaviors with increasing age in male offspring. Brain Res Bull. 2012; 87:295-302.

26. Oh BC, Yeon JY, Lee HS, Lee DW, Park EC. Correlation between private education costs and parental depression in South Korea. BMC Public Health. 2020; 20:972.

27. Ratajska A, Glanz BI, Chitnis T, Weiner HL, Healy BC. Social support in multiple sclerosis: Associations with quality of life, depression, and anxiety. J Psychosom Res. 2020; 138:110252.

28. Zou R, Xu X, Hong X, Yuan J. Higher Socioeconomic Status Predicts Less Risk of Depression in Adolescence: Serial Mediating Roles of Social Support and Optimism. Front Psychol. 2020; 11:1955.

29. Al Omari O, Al Sabei S, Al Rawajfah O, Abu Sharour L, Aljohani K, Alomari K, et al. Prevalence and Predictors of Depression, Anxiety, and Stress among Youth at the Time of COVID-19: An Online Cross-Sectional Multicountry Study. Depress Res Treat. 2020; 2020:8887727.

30. Nicolini H. Depression and anxiety during COVID-19 pandemic. Cir Cir. 2020; 88:542-7.

31. Pahlavanzadeh S, Abbasi S, Alimohammadi N. The Effect of Group Cognitive Behavioral Therapy on Stress, Anxiety, and Depression of Women with Multiple Sclerosis. Iran J Nurs Midwifery Res. 2017; 22:271-5.

32. Yang Z, Oathes DJ, Linn KA, Bruce SE, Satterthwaite TD, Cook PA, et al. Cognitive Behavioral Therapy Is Associated With Enhanced Cognitive Control Network Activity in Major Depression and Posttraumatic Stress Disorder. Biol Psychiatry Cogn Neurosci Neuroimaging. 2018; 3:311-9.

Table 1 Basic characteristics of 1056 pregnant women in Guizhou Province ${ }^{1}$ 


\begin{tabular}{|c|c|c|c|c|c|}
\hline Characteristic & Depression & Depression & Control & Total & $P$ \\
\hline Characteristic & Depression & Depression & Control & Total & $P$ \\
\hline \multicolumn{6}{|l|}{ Age (yrs) } \\
\hline$<18$ & $1(0.13)$ & $1(0.13)$ & $3(1.05)$ & 4 & 0.01 \\
\hline $18-25$ & $170(22.04)$ & $170(22.04)$ & $87(30.52)$ & 257 & \\
\hline $26-30$ & $316(40.99)$ & $316(40.99)$ & $103(36.14)$ & 419 & \\
\hline $31-40$ & 269 (34.89) & $269(34.89)$ & $88(30.88)$ & 357 & \\
\hline $41-50$ & $15(1.94)$ & $15(1.94)$ & $4(1.40)$ & 19 & \\
\hline Residence during the outbreak & Residence during the outbreak & & & & \\
\hline City & $480(62.26)$ & $480(62.26)$ & $141(49.47)$ & 621 & 0.001 \\
\hline Countryside & $268(34.76)$ & $268(34.76)$ & $132(46.32)$ & 400 & \\
\hline Others & $23(2.98)$ & $23(2.98)$ & $12(4.21)$ & 35 & \\
\hline \multicolumn{6}{|l|}{ Education level } \\
\hline Primary school & $16(2.08)$ & $16(2.08)$ & $6(2.11)$ & 22 & 0.014 \\
\hline Junior middle school & $159(20.62)$ & $159(20.62)$ & $84(29.47)$ & 243 & \\
\hline High school & $95(12.32)$ & $95(12.32)$ & $38(13.33)$ & 133 & \\
\hline University and above & $501(64.98)$ & $501(64.98)$ & $157(55.09)$ & 658 & \\
\hline \multicolumn{6}{|l|}{ Primipara } \\
\hline Yes & $398(51.62)$ & $398(51.62)$ & $136(47.72)$ & 534 & 0.260 \\
\hline No & $373(48.38)$ & $373(48.38)$ & $149(52.28)$ & 522 & \\
\hline Monthly household income (yuan) & Monthly household income (yuan) & & & & \\
\hline$<2000$ & $72(9.34)$ & $72(9.34)$ & $42(14.74)$ & 114 & 0.011 \\
\hline $2000-5000$ & $257(33.33)$ & $257(33.33)$ & $109(38.25)$ & 366 & \\
\hline $5000-8000$ & $197(25.56)$ & $197(25.56)$ & $62(21.75)$ & 259 & \\
\hline$>8000$ & $245(31.78)$ & $245(31.78)$ & $72(25.26)$ & 317 & \\
\hline \multicolumn{6}{|l|}{ Pregnancy stage ${ }^{2}$} \\
\hline $1^{\text {st }}$ trimester & $193(25.03)$ & $193(25.03)$ & $65(25.19)$ & 258 & 0.709 \\
\hline $2^{\text {nd }}$ trimester & $344(44.62)$ & $344(44.62)$ & $134(28.03)$ & 478 & \\
\hline $3^{\text {rd }}$ trimester & $234(30.35)$ & $234(30.35)$ & $86(26.88)$ & 320 & \\
\hline \multicolumn{6}{|l|}{ Pregnancy complications ${ }^{3}$} \\
\hline Yes & $153(19.84)$ & $153(19.84)$ & $57(20.00)$ & 210 & 0.955 \\
\hline No & $618(80.16)$ & $618(80.16)$ & $228(80.00)$ & 846 & \\
\hline
\end{tabular}

1 Values are frequencies (

2 The first routine ultrasound examination, $1^{\text {st }}$ trimester; gestational weeks $20-22,2^{\text {nd }}$ trimester; gestational weeks $33-35,3^{\text {rd }}$ trimester.

${ }^{3}$ Pregnancy complications include cardiovascular disease, blood system disease, respiratory system disease, nervous system disease.

Table 2 Correlation coefficient matrix of social support, stress and depression $(\mathrm{n}=1056)$

\begin{tabular}{llll}
\hline Variables & Depression & Social support & Stress \\
\hline Depression & 1.00 & & \\
Social support & $-0.40^{* *}$ & 1.00 & \\
Stress & $0.27^{* *}$ & $-0.34^{* *}$ & 1.00 \\
\hline
\end{tabular}


$* *: p<0.01$

Table 3 Pathway coefficients of structural equation model $(\mathrm{n}=1056)$

\begin{tabular}{llllll}
\hline Pathways & Estimate & Std. Estimate & SE & z & $P$ \\
\hline Social support- Depression & -0.09 & -0.34 & 0.01 & -8.47 & $<0.001^{* *}$ \\
Stress- Depression & 0.22 & 0.50 & 0.02 & 9.86 & $<0.001^{* *}$ \\
Social support-Stress & -0.06 & -0.09 & 0.02 & -2.71 & $0.007^{* *}$ \\
\hline
\end{tabular}

Std. Estimate Standardized estimate, SE Standard error

$* *: p<0.01$

Table 4 Coefficients of measurement model and structural model among pregnant women $(\mathrm{n}=1056)$

\begin{tabular}{llllll}
\hline Factor loadings & Estimate & Std. Estimate & SE & z & $P$ \\
\hline Stress & & & & & \\
Coping with stress & 0.95 & 0.69 & 0.04 & 22.44 & $<0.001^{* *}$ \\
$\begin{array}{l}\text { Perceived stress } \\
\text { Social support }\end{array}$ & 1.12 & 0.79 & 0.04 & 26.1 & $<0.001^{* *}$ \\
Family support & 1.15 & 0.85 & & & \\
Friends support & 1.13 & 0.88 & 0.04 & 29.82 & $<0.001^{* *}$ \\
Important people support & 1.08 & 0.81 & 0.04 & 30.89 & $<0.001^{* *}$ \\
\hline
\end{tabular}

Std. Estimate Standardized estimate, SE Standard error

$* *: p<0.01$

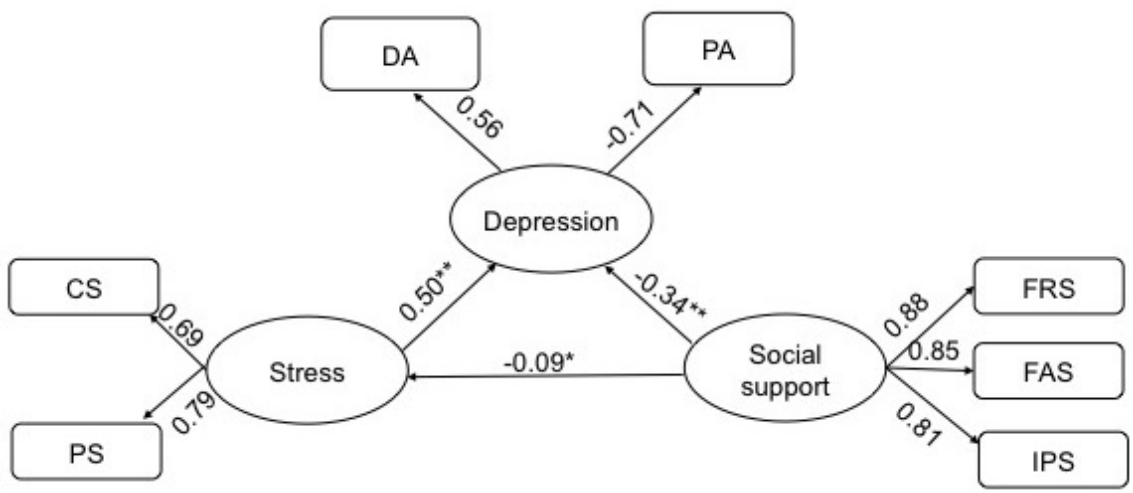

